\title{
Siedlungsgeographie und Agrarlandschaften Europas
}

\author{
Werner A. Gallusser
}

Zum «Symposium international de géographie de l'habitat et des paysages agraires d'Europe», Liège

Vom 29. Juni bis 5. Juli 1969 waren in Lüttich rund 70 Siedlungs- und Agrargeographen aus elf Staaten Europas zum 5. Fachsymposium vereinigt.

Eine dreitägige Exkursion, von Dozenten der Universitäten Lüttich, Löwen und Gent vorzüglich geleitet, legte einen instruktiven Querschnitt durch belgische Siedlungsräume. Am ersten Tag wurde das Gebiet Lüttich-Gent besucht, und zwar zuerst das Verstädterungsgebiet von Lüttich, die angrenzenden lößreichen Agrarlandschaften des Hesbaye (Xhendremael - Oleye - Hasselt) und die sehenswerte Domäne Boekrijk, ein Freilichtmuseum für Bauernhäuser, das in Planung und Ausführung weitgehend den belgischen Geographen zu verdanken ist. Der Nachmittag war der Bergbau- und Agrarlandschaft der Campine gewidmet (Zonhoven-BeringenGeel-Herentals), wo landwirtschaftliche Neusiedlungen und aktuelle Umbauten der Dörfer studiert werden konnten. Gegen Abend wurde die Scheldeniederung und der Ubernachtungsort Gent erreicht.

Einen ersten Höhepunkt des zweiten Tages bot die Besichtigung einiger hervorragender Denkmäler flandrischer Kunst in Gent (Belfried, van-EyckFlügelaltar in der St.-Bavo-Kathedrale); ebenso eindrücklich erschienen uns die Beispiele aus der mittelalterlichen Kulturlandschaftsgeschichte im Bereich der flandrischen Exkursionsstrecke (Lambeke -Drongen-Munkzwahlm). Am Standort Munkzwahlm konnte auf Grund ausgezeichneter Quellen und Planunterlagen die Genese einer regionaltypischen «Kouter»-Siedlung erklärt werden. Die anschließende Fahrt durch das sogenannte Pajottenland (im westlichen Umland von Brüssel) führte über Liedekerke-Borchtlombeek nach Gooilk. Sie zeigte die Anpassung eines Agrarraumes an die Bedingungen der benachbarten Großstadt Brüssel: Kleinlandwirtschaft mit Intensivkulturen und einen ausgeprägten Pendelverkehr. Dazu zwei bleibende Eindrücke: die Massen von Velos und Autos am Pendlerbahnhof von Liedekerke und die Erdbeerernte in Borchtlombeek, dem Zentrum des bajottenländischen Erdbeer- und Himbeeranbaus (auf weite Strecken hatten die Erdbeerverkäufer ihre Verkaufsstellen längs der Autostraße errichtet). Vom bisher flachen Tiefland wechselte man danach - zwischen Halle und Nivelles - in die schwach gewellte Landschaft des Brabanter Plateaus, deren Fruchtbarkeit in den großflächigen Zuckerrübenund Weizenkulturen zum Ausdruck kommt. In Namur endete die zweite Tagesfahrt.

Die erste Strecke des dritten Tages führte über Bois de Villers-Graux-Bioul durch die Marlagne, eine vorwiegend im Carbonkalk angelegte Gebirgslandschaft, deren Bevölkerung landwirtschaftlich und, infolge der Pendelwanderung nach den Industrieorten der Sambre- und Meusetalung, auch industriell geprägt ist. Im Gegensatz zu den nördlichen Gebieten mit den Backsteinbauten beobachteten wir hier das meist immer noch zu Straßenzeilen gefügte Steinhaus. Bei Dinant gelangte man wieder in das Meusetal und durchfuhr danach über CellesCustinne-Ciergnon die Kalkschieferzone der Famenne schisteuse. Die Siedlungen gehörten nun zunehmend zum Typ der Haufendörfer und -weiler; Wald und Grünland breiteten sich mit ansteigender Meereshöhe stärker aus. Focant, ein Dorf von ca. 315 Einwohnern, bot ein vorzügliches Beispiel für die neuzeitliche Flurentwicklung: ausgedehnte Gemeindeländereien wurden unter dem wachsenden Bevölkerungsdruck des 19. Jahrhunderts - wie vielerorts in Europa - in regelmäßige Feldparzellen aufgeteilt («Allmendaufteilungsflur»). Diese werden noch immer alle 9-18 Jahre an die Nutzungsberechtigten neu verliehen. Die vorwiegend der Futterproduktion dienenden Flächen bildeten mit ihrer klar erkennbaren schematischen Parzellierung ein markantes Element der hiesigen Agrarlandschaft. Bei Halma erreichten wir den Úbergang zu den Ardennen. Dieses wald. und weidereiche Gebirgsland querten wir auf der Strecke St. HubertOrtho-Baraque de Fraiture. Allgemein war hier bei regressiver Landwirtschaft ein Fortschreiten des Fremdenverkehrs festzustellen. Oft standen die quarzitgemauerten Einhaus-Bauernhöfe leer, oder sie dienten etwas modernisiert der erholungssuchenden Bevölkerung aus dem belgischen Industriegebiet. Auf der Fahrt von Bra-Stoumont-Verviers gegen die Verstädterungszone von Lüttich hin konnten wir die zunehmende landschaftliche Differenzierung der Wohn- und Erholungsfunktion einprägsam erleben.

Die Arbeitssitzungen des Symposiums fanden vom 2. bis 4. Juli im Séminaire de Géographie der Uni- 
versität Lüttich statt. Unter wechselnder Leitung der Damen Prof. A. Krenzlin, Frankfurt, und Dr. E. Gotschalk, Amsterdam, sowie der Herren Professoren und Dozenten R. Buchanan, Belfast, C. Christians, Liège, S. Dahl, Göteborg, A. Felrei, Clermont-Ferrand, wurden an sechs Halbtagssitzungen 25 Studienberichte vorgelegt und diskutiert. Je vier Studien waren belgischen, deutschen und französischen Untersuchungsgebieten gewidmet; ein Referat war rein methodisch ausgerichtet, und die restlichen verteilten sich auf Untersuchungsräume in elf weiteren Ländern Europas. Gegenüber dem Würzburger Symposium war eine merkliche Verschiebung der Thematik von der ausgesprochen «historischen» Siedlungsgeographie zur "aktualdynamischen" festzustellen, wobei eine Reihe von Arbeiten in klassischer Weise versuchten, die landschaftlichen Entwicklungsgänge jeweils vom Mittelalter oder zumindest von der Frühneuzeit her bis zur Gegenwart nachzuzeichnen.

Weil die wissenschaftlichen Beiträge später in einem Symposiumsband publiziert werden, beschränken wir uns hier auf eine kurze Mitteilung der Themen nebst einigen persönlichen Kommentaren. Folgende Referenten befaßten sich mit siedlungs- und agrargeographischen Themen aus geschichtlich zurückliegenden Zeiträumen:

R. A. Butlin, Dublin: Irish Agrarian History; review and prospect.

X. de Planhol, Paris: Aux origines de l'habitat rural lorrain.

H. Grees, Tübingen: Das Kleinbauerntum in Ostschwaben und sein Einfluß auf die Entwicklung von Siedlungen und Wirtschaft.

A. Harris, Hull, und W. Matzat, Frankfurt: Preenclosure parcel patterns and field systems in the East Riding of Yorkshire.

G. R. J. Jones, Leeds: The federal estate as a model framework for tracing early stages in the evolution of rural settlement.

J. Peltre, Nancy: Les faits d'orientation dans la structure agraire en Lorraine.

F. Snacken, Gent: Les champs bombés du Pays de Waas.

Die Studie von H. Grees über das ostschwäbische Seldnertum darf als ein vorzüglicher Beitrag zum Problem der südwestdeutschen Anerbengebiete bezeichnet werden. Auch für unsere Schweizer Verhältnisse erhalten die Erkenntnisse des Tübinger Geographen ein besonderes Gewicht, weil er auf Grund seiner Quellenstudien die sozialgeographische Entwicklung in Anerbenräumen zu differenzieren vermag. Gerade das recht komplexe Nebeneinander von Erbbauern auf Vollerwerbsstellen und den vielfältigen Formen des Kleinbauerntums sowie die räumliche Verteilung dieser Sozialgruppen im dörflichen Siedlungsbild und Grundbesitz (Allmendproblem) erfahren dadurch eine wissenschaftliche Klärung. J. Peltre konnte anhand von Zusammenlegungsbeschrieben in Flurbüchern des 17. Jahrhunderts belegen, daß in zahlreichen Gemeinden südöstlich von Metz die Gewanne nach zwei Hauptachsen (NNW-SSE; WNW-ESE) orientiert erschienen. Weil das Flachrelief des Untersuchungsgebietes keine natürliche Erklärung für eine derartige Orientierung ableiten läßt, bevorzugt Peltre eine kulturelle Begründung: er nimmt an, die Parzellenorientierung hänge in diesem keltorömisch besiedelten Raum mit einer früheren - vielleicht religiös bedingten - Siedlungsausrichtung zusammen, eine Annahme, welcher in der regen Diskussion mit dem Argument begegnet wurde, auch neuzeitliche $\mathrm{Zu}$ sammenlegungen erbrächten als rationale Maßnahme oft eine einheitliche Parzellenorientierung.

Der Berichterstatter faßt als eine zweite thematische Einheit jene Vorträge zusammen, welche die heutigen Siedlungsverhältnisse aus einem historischen Rückgriff - meist bis ins 18. oder 19. Jahrhundert zurück - entwickelnd darstellen:

B. Freund, Frankfurt: Siedlungsgenetische Untersuchungen in der Terra de Borroso.

F. Scholz, Karlsruhe: Veränderungen in der Agrarlandschaft des nordöstlichen Schwarzwaldes unter dem Einfluß von Industrie und Fremdenverkehr.

G. Schwarz, Freiburg i. Br.: Die Siedlungs- und agrargeographische Entwicklung der oberen Markgrafschaft im Bereich von Aue und Niederterrasse seit dem Ende des 18. Jahrhunderts.

R. Zschocke, Aachen: Die Entwicklung der landwirtschaftlichen Betriebsgrößen in den Rheinlanden und ihre Auswirkung auf die Kulturlandschaft.

Die Studie von B. Freund über eine nordportugiesische Kulturlandschaft bereicherte das europäische Symposium auch in regionaler Hinsicht. Aus der Fülle der erarbeiteten siedlungsgeographischen Befunde heben wir zwei Tatbestände hervor, welche die besondere Situation portugiesischer Landschaftsentwicklung erhellen. Der erste widerspricht der Behauptung, "dass Reste einer vorrömischen kollektivistischen Agrarverfassung hier tradiert seien. Die als typisch geltenden Merkmale wie egalitäre Landanteile an der Allmend, Zelgenbindung, dorfgemeinschaftliche Rodungen usw. erwiesen sich als relativ jung". Des weiteren vollzogen sich hier bedeutende Veränderungen der Landnutzung, wie die Verbesserung der Zelgenwirtschaft durch den Hackfruchtbau, Aufforstungen und Allmendteilungen, erst in den letzten drei Jahrzehnten, worin sich eine ausgeprägte Retardierung gegenüber entsprechenden Vorgängen in Mitteleuropa (um 1800) widerspiegelt. 
Die aktuelle landschaftliche Dynamik scheint die Geographen immer wieder neu und in zunehmendem Maße zur Untersuchung anzuregen. $\mathrm{Da}$ sich dieses Arbeitsfeld als ausnehmend fruchtbar erweist, zeigt die Vielfalt der methodischen Ansätze. Zudem muß es jeden Geographen mit Zuversicht erfüllen, wenn er gerade bei Gelegenheit eines internationalen Symposiums feststellen darf, daß der "Aufbruch» zur aktualgeographischen Forschung in den meisten Ländern sozusagen spontan erfolgt ist. So bezogen sich die folgenden Referate dieses Problembereichs auf Regionen aus acht verschiedenen Staaten West- und Osteuropas:

A. R. H. Baker, Cambridge: Some shape and contact characteristics of french rural communes.

A. Beuermann, Braunschweig: Junge Siedlungsformen in Griechenland.

H. Desplanques, Lille: L'influence urbaine sur les paysages ruraux en Italie centrale.

F. Dussart und C. Fourez, Liège: Les secondes résidences en Ardenne; le cas de la commune de la Gleize.

G. Enequist, Uppsala: Holdings and population in the rural districts of Sweden in the 20th Century.

W. Gallusser, Basel: Auswirkungen des Autobahnbaues auf die Agrarstruktur ländlicher Räume, dargelegt am Beispiel der Nordwestschweiz.

W. Grillet, Leuven: Paysage et milieu rural en évolution: la Campine. Urbanisation d'un milieu rural? S. Helmfrid, Stockholm: Stockholmer Kulturlandschaftsforschung. Eine Übersicht laufender Projekte.

S. Ilesic, Ljubljana: Die neuesten Umbildungen der traditionellen Agrarlandschaft Sloweniens.

H. Keeris, Gent: Evolution récente du paysage rural dans la région frontière de la campine belgeneerlandaise.

M. Kielczewska-Zaleska, Warschau: Le remembrement des champs en Pologne après la seconde guerre.

R. Livet, Aix-en-Provence: Quelques exemples d'urbanisation des campagnes dans le Sud-Est français. S. Zajchowska, Poznan: Problèmes d'urbanisation de la campagne en Posnanie.

Sowohl für die historische Geographie als auch für die Planung boten die Ausführungen Bakers wertvolle methodische Anregungen. In Anlehnung an ein von Haggett (1965) entwickeltes Berechnungsverfahren untersuchte Baker gegen 300 französische Landgemeinden nach ihren Umrißformen. Es galt festzustellen, ob für die ländlichen Gemeinden das Sechseckmodell vorherrschend sei. Dabei interessiert geographisch weniger die Form an sich als die darin zum Ausdruck kommenden Kontaktmöglichkeiten zu den Nachbargemeinden. Tatsächlich hat die Gestaltanalyse keine Vorherrschaft des Hexagons erbracht, hingegen - durch eine ergänzende
Abklärung der «Kontaktzahlen» - eine mittlere Kontaktzahl von 6,21. Die in der lebhaften Diskussion umstrittene Methode dürfte in bezug auf die reine Formanalyse kaum neue Gesichtspunkte ergeben, denn die linienmäßige Grenzführung wird von zu vielen naturräumlichen und politisch-territorialen Faktoren bestimmt; uns erscheint jedoch die systematische Untersuchung der zwischengemeindlichen Kontaktstellen, wie sie Baker versucht hat, als ein fruchtbarer Ansatz zu weiteren Erkenntnissen.

Eine Reihe von Studien setzte sich insbesondere mit der Urbanisierung des ländlichen Raumes auseinander: so differenzierte Livet für SE-Frankreich einige bestimmte Kriterien zur Erfassung des Urbanisierungsgrades, wogegen Desplanques für Nordumbrien in eindrücklicher Klarheit ableitete, daß neben der aktuellen Verstädterungsbewegung auch eine historisch bedingte Art von "Verländlichung" der Stadt (Perugia) insofern bestehe, als hier schon seit dem Mittelalter viele städtischen Grundbesitzer mit ausgedehnten Ländereien verbunden seien. Diese städtischen Landeigentümer bewahrten ihre traditionelle Bindung zur Landwirtschaft und eine gewisse rustikale Gesinnung bis heute. Am Beispiel der Region Posen erläuterte Frau Zajchowska das Hauptmerkmal wachsender Urbanisierung ursprünglicher Agrargebiete: "Le pourcentage de population non agricole doit être considéré comme mesure principale du processus d'urbanisation, car l'accroissement du pourcentage de ce groupe de population est stimulateur de changements ultérieurs d'autres éléments de la structure sociale et économique.»

Schließlich verdient die Übersicht von S. Helmfrid über das Forschungsprogramm der Stockholmer Kulturgeographen eine kurze Würdigung, bestätigt sie doch die schon erwähnte internationale Tendenz, im Rahmen der Kulturgeographie zielbewußt auch die Probleme des rezenten Landschaftswandels anzugehen, ohne die eigentliche Kulturlandschaftsgeschichte $\mathrm{zu}$ vernachlässigen. Das von einem erfreulichen Optimismus getragene «Stockholmer Programm» gliedert sich in folgende drei Aufgabenbereiche:

1. Die historische Kulturlandschaftsforschung unter D. Hannerberg;

2. Die subrezente Kulturlandschaftsgeschichte unter Leitung von S. Helmfrid, welche die Landschaft unmittelbar vor dem industriezeitlichen Umbruch untersucht (i. d. R.: 19. Jahrhundert);

3. Die geographische Analyse des rezenten Kulturlandschaftswandels unter S. O. Lindquist.

Es ist zu hoffen, daß den Stockholmer Kollegen der Plan gelingen möge, ein besonderes Institut für Kulturlandschaftsforschung zu errichten. In diesem In- 
stitut könnten wichtige Grundlagen zur besseren Kenntnis des landschaftlichen Milieus erarbeitet werden. Angesichts der drängenden Probleme des menschlichen Lebensraumes wäre es von allgemeinem Interesse, daß das schwedische Experiment gelänge.

Der theoretisch fundierte Vortrag von C. Lienau, Gießen, über den «Entwurf eines terminologischen Rahmensystems für die Erfassung funktionaler Siedlungstypen des ländlichen Raumes» beendete das Symposium; thematisch bildete er den Auftakt zur anschließenden Versammlung der "Internatio. nalen Arbeitsgruppe für die Terminologie der Agrarlandschaft» vom 5. Juli unter Leitung von Prof. $\mathrm{H}$. Uhlig, Gießen. Nachdem die Ergebnisse der bisherigen Arbeit über die Flurformen in einem ersten Band vorliegen, können aus der sich daraus entwickelnden internationalen Diskussion Erfahrungen für die weiteren Aufgaben gewonnen werden. Als nächstes Sachgebiet sollen die ländlichen Siedlungen behandelt werden, über welche einleitend der Entwurf eines terminologischen Rahmensystems vorgelegt werden konnte. In einer einläßlich geführten Diskussion bereinigten die Mitglieder der Arbeitsgruppe die theoretischen Grundzüge dieses Rahmensystems.
Während des Symposiums wurde in den Räumen des Geographischen Seminars eine Ausstellung über belgische Siedlungs- und Agrarlandschaften gezeigt. Die reich dokumentierte Schau vermittelte ein umfassendes Bild sowohl von der intensiven Arbeit der belgischen Geographen wie auch von der ansehnlichen Stellung, welche die Geographie in Belgien einnimmt. Davon zeugten auch die großzügig offerierten Empfänge der Symposiumsteilnehmer durch den Rektor der Universität Lüttich, den Bürgermeister der Stadt und durch die Behörden der Province de Liège.

Es verbleibt uns noch, den Lütticher Geographen für den mustergültig organisierten Verlauf des Symposiums zu danken. Daß diese europäische Tagung auch sprachlich in geordneten Bahnen verlaufen konnte, war weitgehend das Verdienst von Frau Dr. Dussart, Liège. Abschließend sei dem Initianten und Organisator des Symposiums, Herrn Prof. F. Dussart, aber auch seinem Kollegen Prof. C. Christians, den weiteren Mitarbeitern des Geographischen Institutes Lüttich sowie allen mitbeteiligten belgischen Geographen ihr erfolgreicher Einsatz für die europäische Siedlungs- und Agrargeographie herzlich verdankt. 